\title{
Research on Road Traffic Network Design Model and Algorithm Considering Traffic Fairness
}

\author{
SiWen Zhao ${ }^{1, *}$ \\ ${ }^{1}$ School of traffic and transportation, Beijing Jiaotong University, Beijing
}

\begin{abstract}
Urban transportation is an important part of urban economic structure, which plays an important role in promoting the orderly development of society, residents' work and life. This paper analyzes the fairness in traffic network design problem, constructs the average network improvement index to measure fairness, and establishes a bi-level programming model for road traffic network design considering traffic fairness. The upper model includes the optimal total travel time and traffic fairness, and the lower model is the traffic flow allocation model. Then, according to the characteristics of the model, a genetic algorithm is designed to solve the model. Finally, a simple example is given to illustrate the effectiveness of the mathematical model and algorithm. The model proposed in this paper can get the final road design scheme satisfying the total travel time and traffic fairness of the road network under certain road network conditions, and can provide decision support for traffic planners.
\end{abstract}

\section{Introduction}

With the rapid development of China's economy and the accelerating process of urbanization, the contradiction between urban traffic supply and demand is increasingly serious. On the one hand, the rapid growth of urban population has brought about an increase in the demand for urban traffic, and the gradual improvement of residents' quality of life, which has put forward higher requirements for urban area and transportation infrastructure. On the other hand, compared with the increasing passenger flow and the rapid development of economic situation, road resources and construction resources to improve road facilities are relatively scarce. In order to meet the growing traffic demand, we must improve the road network and reduce the gap between supply and demand. Therefore, in the limited total amount of resources, how to reasonably allocate road resources has become a major problem faced by traffic policy.

Urban traffic network design problem(UTNDP) is an important part of traffic planning. The core content of its research is to find the optimal construction investment scheme suitable for new construction or improvement of roads or public networks through optimization calculation on the premise that road traffic users meet certain route selection behavior criteria. Therefore, the study of traffic network design is an effective method and measure to solve urban traffic problems. The traditional urban traffic network design problem usually takes the minimum total impedance of the whole road network as the optimization objective. However, the existence of Braess phenomenon indicates that under the premise of increasing the traffic capacity of the road network, the travel time of some travelers may still increase, leading to the deterioration of traffic conditions. Meng and Yang[1] confirmed in continuous network design problems, when the network with minimum total cost, improve the benefit of the selected road traffic capacity will lead to some OD the route of travel costs, they put forward a applicable to transportation network design problem of fairness index, and the fairness into the objective function of the traffic network design. This finding has attracted a lot of attention. In addition, other scholars have established a road network design model considering traffic fairness. Antunes[2], Chen and Yang[3], Santos [4], Barbati[5]et al incorporated spatial equity into the road network design model. However, spatial equity usually involves the population size, social, economic, environmental and other factors of multiple cities. From the macro level, the fairness of regional road network design is considered while individual behavior choices and intuitive feelings are ignored.

Urban traffic network design is of great significance in economic, social and other aspects. Integrating personal perception into the study of traffic network design conforms to the long-term planning of urban construction and is conducive to promoting the harmonious development of cities. The study content of the related literature at home and abroad, this paper puts forward the average network improvement indicators as a fair measure of the traffic, builds the mathematical model of continuous network design based on traffic fair, and according to the characteristics of the mathematical model of the improved genetic algorithm is constructed, and exercise the algorithm to a small network of road network design problems.

\footnotetext{
*Corresponding author: 19120967@bjtu.edu.cn
} 


\section{Model Specifications}

Transportation network design problem is usually expressed in bi-level programming model, from the perspective of traveler, traveler and preferences choose travel path according to your own judgement, as a form of travel information to planners decision-making basis, planners, according to the existing road network under the adjusted travel information of road network, give a road network from the perspective of system, the optimal solution, Travelers then choose the road network according to their preferences again, and do this for many times, and finally get the road network plan that meets the interests of both travelers and planners. In the two-level programming model, the upper model represents the planner's goal and the lower model represents the traveler's goal. In this paper, the objective function of the upper model is the minimum weighted average of the total travel time of the system and the average network improvement index. The lower model adopts user balance (UE) flow distribution model.

In the actual planning and design work, the public's acceptance of a road network improvement scheme largely depends on the income impact of the road network on the individual. After verification, if an optimal network design scheme is implemented, the travel cost of some OD pairs may increase after the road network flow reaches the equilibrium state, it shows that some of the travelers may be damaged because of the upgrading of the road network, and these groups lead to the discussion and Research on traffic fairness.

Let $y_{a}$ denote the capacity improvement of the road section after road section reconstruction, variable $y=\left(\cdots, y_{a}, \cdots\right)^{T}$ can be expressed as a scheme of network design, and the set of all schemes is expressed as:

$$
Y=\left\{\begin{array}{l}
y=\left(\cdots, y_{a}, \cdots\right)^{T} \mid \sum_{a} g_{a}\left(y_{a}\right) \leq B, \\
0 \leq y_{a} \leq u_{a}, a \in A
\end{array}\right\}
$$

where: $g_{a}\left(y_{a}\right)$ represents the construction investment function of capacity improvement. $B$ is construction investment budget. $u_{a}$ is upper bound of decision variables. $A$ is the collection of road sections on the transportation network.

In order to quantitatively represent the equity change of each traveler before and after the network scheme change, the average network improvement indicators are defined as follows:

$$
\alpha(y)=\sum_{r s \in W} \frac{\mu_{r s}(y)}{\overline{\mu_{r s}}}
$$

where: $r s$ represents the starting node and end point of a trip. $W$ represents the set of OD pairs. $\mu_{r s}(y)$ represents the travel impedance of OD pairs against the balanced distribution current between $r s$ after the road network is improved. $\bar{u}_{r s}$ represents the travel impedance of OD pair $r s$ under balanced distribution before the road network improvement. $\frac{\mu_{r s}(y)}{\mu_{r s}}$ reflects the fairness change between OD pair $r s$ before and after road network improvement.

$\sum_{r s \in W} \frac{\mu_{r s}(y)}{\overline{\mu_{r s}}}$ represents the overall fairness of multiple OD pairs in the whole network. If $\sum_{r s \in W} \frac{\mu_{r s}(y)}{\overline{\mu_{r s}}}>1$, it means that the road network scheme change worsens the fairness of OD pair $r s$ to travelers. If $\sum_{r s \in W} \frac{\mu_{r s}(y)}{\overline{\mu_{r s}}}=1$, it means that the change of road network scheme has no effect on the traffic fairness of OD pair $r s$.If $\sum_{r s \in W} \frac{\mu_{r s}(y)}{\overline{\mu_{r s}}}<1$, it means that the road network scheme changes to improve the fairness of traffic between OD pair rs. In the model, in order to ensure that the change of road network scheme can improve the traffic fairness of each traveler, Let $\alpha(y) \leq \beta$, and the value of $\beta$ is set by the planner, $\beta$ as a fairness constraint, it is usually less than or equal to 1.

The bi-level programming model is established, in the upper layer of the model, the weighted average value of the total travel time and fairness of the system is minimized. In the lower layer of the model, we assume that travelers can always accurately obtain the traffic situation of the whole road network and choose the travel path with less travel time according to their own judgment. The lower layer of the model is the classic user equilibrium traffic assignment model.

The upper model is:

$$
\min _{y} F(y)=w_{1} \sum_{a \in N} t_{a}\left[x_{a}(y), y_{a}\right] x_{a}(y)+w_{2} \alpha(y)(3)
$$

s.t.

$$
\begin{gathered}
\alpha(y) \leq \beta \\
\sum_{a} g_{a}\left(y_{a}\right) \leq B \\
0 \leq y_{a} \leq u_{a}
\end{gathered}
$$

where: $t_{a}\left[x_{a}(y), y_{a}\right]$ represents the impedance function of road $a \cdot x_{a}(y)$ represents the flow of $\operatorname{road} a \cdot \beta$ represents upper fair value. $w_{1}, w_{2}$ represents weight parameter.

$x_{a}(y)$ is obtained by the flow distribution of the lower model, which has the following form:

$$
\min \sum_{a \in A} \int_{0}^{x_{0}} t_{a}\left[x_{a}(y), y_{a}\right] d x
$$

s.t.

$$
\sum_{p \in P_{r}} f_{r s}^{p}=q_{r s}, \forall r s \in W
$$




$$
\begin{gathered}
x_{a}=\sum_{r s \in W} \sum_{p \in r s} \delta_{r s}^{a p} f_{r s}^{p}, \forall a \in A, r s \in W \\
f_{r s}^{p} \geq 0, \forall p \in P_{r s}, r s \in W
\end{gathered}
$$

where: $x_{a}$ represents the flow of $\operatorname{road} a \cdot \delta_{r s}^{a p}$ is associated variables of path and road section, if road $a$ is used in path $p$, it is equal to 1 , otherwise it is equal to $0 . f_{r s}^{p}$ represents the traffic flow on any path $p$ between OD pair $r s . q_{r s}$ represents the traffic flow between OD pair $r s . P_{r s}$ represents the set of all paths between OD pair $r s$.

\section{Algorithm design}

UTNDP is a bi-level game in concept, which includes two different types of games, namely leader-follower game and non-cooperative Nash game. Due to the NP-hard nature of bi-level programming problem, it is very difficult to solve it by exact solution. The bi-level programming model is non-convex. Even if the upper and lower level problems are convex, the convexity of bi-level programming can not be guaranteed.

The UTNDP solution includes accurate mathematical solution and heuristic solution. Accurate methods such as branch delimitation, branch backtracking or mathematical planning technology rely on mathematical properties to solve problems, so that they can reach local optimal at least. But most of the solutions are not suitable for medium or large networks. In this paper, the improved genetic algorithm is used to solve the model. Compared with the general mathematical solution, its advantages are strong search ability and expansibility, which can avoid the algorithm from falling into local optimal by mutation mechanism. The disadvantage is that some parameters need to be determined by experience and can not guarantee the global optimization.

The genetic algorithm is designed as follows:

Step1: Initialize the road network, generate the initial road network scheme $y_{0}$, set the population number $N$, and according to the decision variable $y_{a}$, the initial population $P_{0}$ is generated randomly under the restriction of $u_{a}$.

Step2: The MSA algorithm is used to allocate users to each chromosome $\mathrm{Y}_{a}$ in the population, and the total travel cost and average network improvement index of each chromosome $\mathrm{y}_{a}$ are obtained.

Step3: The travel cost formed by each chromosome $\mathrm{y}_{a}$ and the average network improvement index data are standardized, and the fitness function $C$ is calculated.

Step4: According to fitness function $C$, the population is selected, and the individuals with poor adaptability are eliminated to generate population $P_{1}$.

Step5: Set cross probability $p_{\mathrm{c}}$. The chromosome was selected according to probability to cross operation. The population $P_{2}$ was obtained by randomly generating cross point position and cross chromosome.
Step6: Set the mutation probability $p_{z}$, and select the chromosome mutation operation according to the probability. The mutation points were randomly generated to obtain population $p_{3}$.

Step7: Screen out the chromosome $\mathrm{y}_{a}$ that does not meet the construction constraint $B$, and generate the chromosome population that meets the conditions. population $P_{4}$ was obtained.

Step8: Return to Step2, the number of iterations $n=n+1$. When $n$ meets the number of iterations, execute step2 and step3, and then go to step9.

Step9: Stop genetic algorithm, select the chromosome with the minimum fitness function in population $P_{4}$, that is the best fitness, and input the corresponding scheme of the chromosome, that is the optimal road network design scheme.

\section{Case analysis}

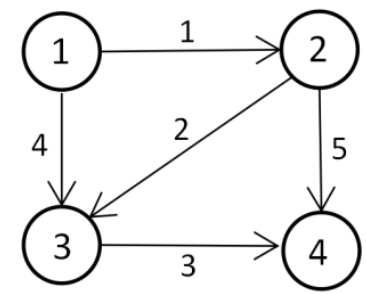

Fig. 1. A simple road network diagram

Fig. 1 is a simple road network with two OD pairs, $q_{14}$ $=200, q_{23}=300$ are the traffic demand of the network. The road impedance follows the formula of:

$$
t_{a}=x_{a}\left(1+0.1\left(\frac{x_{a}(y)}{C_{a}+y_{a}}\right)^{2}\right)
$$

Parameters of each road section:

Table 1. Parameters of each road section

\begin{tabular}{|c|c|c|}
\hline$a$ & $x_{a}$ & $C_{a}$ \\
\hline 1 & 2 & 80 \\
\hline 2 & 5 & 150 \\
\hline 3 & 3 & 100 \\
\hline 4 & 4 & 120 \\
\hline 5 & 3 & 80 \\
\hline
\end{tabular}

The cost of unit flow for road expansion is $[50,60,80,40,20]$ thousand yuan, and the budget constraint is 4 million yuan. Maximum road expansion $u_{a}=30$.

After repeated debugging, the average network improvement index weight $w_{1}=0.3$, the total travel time weight $w_{2}=0.7$, the crossover probability $p_{c}=0.6$, the 
mutation probability $p_{z}=0.2$, and the number of iterations $n=10$.

Because the genetic algorithm is affected by the initial population and other parameters, this paper sets different initial population numbers to compare the results. According to the fitness function, only the total cost of the road network is considered. The results are shown in Table 2. The weighted fitness of the optimal result is calculated. When the population number is 300 , the fitness is optimal. Total cost of the road network is 3246.68 , the average network improvement index is 0.9417 , and the corresponding road network expansion scheme is $[11,23,16,3,27]$.

Table 2. Results based on general road network design model.

\begin{tabular}{|c|c|c|c|}
\hline $\begin{array}{c}\text { Population } \\
\text { size }\end{array}$ & $\begin{array}{c}\text { Total cost } \\
\text { normalization }\end{array}$ & $\begin{array}{c}\text { Fair value } \\
\text { normalization }\end{array}$ & Fitness \\
\hline 10 & 1 & 0.39 & 0.82 \\
\hline 50 & 0.88 & 1 & 0.91 \\
\hline 100 & 0.48 & 0.73 & 0.56 \\
\hline 200 & 0.18 & 0.44 & 0.26 \\
\hline 300 & 0.08 & 0 & 0.05 \\
\hline 400 & 0.29 & 0.56 & 0.37 \\
\hline 500 & 0 & 0.22 & 0.07 \\
\hline
\end{tabular}

Table 3 shows the results obtained according to the model in this paper. It can be seen that when the population number is 400 , the fitness is the best. At this time, the total cost of the road network is 3230.58 , and the average network improvement index is 0.9410. After normalization, according to the weight, the final fitness is the smallest. When the fitness reaches the optimal condition, the corresponding road network expansion scheme is $[10,24,17,2,28]$.

Table 3. Results of road traffic network design model considering traffic fairness.

\begin{tabular}{|c|c|c|c|}
\hline $\begin{array}{c}\text { Population } \\
\text { size }\end{array}$ & $\begin{array}{c}\text { Total cost } \\
\text { normalization }\end{array}$ & $\begin{array}{c}\text { Fair value } \\
\text { normalization }\end{array}$ & Fitness \\
\hline 10 & 0.55 & 1.00 & 0.69 \\
\hline 50 & 1.00 & 0.76 & 0.93 \\
\hline 100 & 0.69 & 0.23 & 0.55 \\
\hline 200 & 0.66 & 0.15 & 0.51 \\
\hline 300 & 0.55 & 0.00 & 0.38 \\
\hline 400 & 0.00 & 0.29 & 0.09 \\
\hline 500 & 0.27 & 0.44 & 0.32 \\
\hline
\end{tabular}

From the comparison of the two results, it can be seen that the calculation results of the road traffic network design model considering traffic equity are better in the total cost of road network and the average network improvement index. The results show that the algorithm is more reasonable, more fully considered, and has practicability and effectiveness.

\section{Conclusion}

Urban traffic network design is of great significance in economic, social and other aspects, and the study of fairness in traffic network design will help to build a harmonious city and promote the harmonious development of human and society. This paper constructs a road traffic network design model considering traffic fairness, fully considers the fairness improvement degree of each OD pair after the road network expansion, and improves the genetic algorithm on this basis. Finally, an example is given to verify the feasibility and effectiveness of the model and algorithm. It provides a new idea for the research of fairness in road network design, and is helpful for the further research of fairness in road network design in the future.

\section{References}

1. Meng Q, Yang H. Benefit distribution and equity in road network design[J]. Transportation Research Part B: Methodological, 36(1), 19-35,(2002)

2. Antunes A, Seco A, Pinto N. An accessibilitymaximization approach to road network planning,Computer-Aided Civil and Infrastructure Engineering, 18(3),224-240 (2003)

3. Chen A, Yang C. Stochastic transportation network design problem with spatial equity constraint[J]. Transportation Research Record, 1882(1),97-104 (2004)

4. Santos B, Antunes A, Miller E J. Integrating equity objectives in a road network design model[J]. Transportation Research Record, 2089(1),35-42 (2008)

5. Barbati M. The design of transportation networks: a multi objective model combining equity, efficiency and efficacy, OASIcs: Open Access Series in Informatics, 22,75-83 (2012) 